\title{
EAGLE multi-object AO concept study for the E-ELT
}

\author{
G. Rousset ${ }^{1, \text { a }}$, T. Fusco ${ }^{2}$, F. Assemat ${ }^{1,2}$, T. Morris ${ }^{3}$, E. Gendron ${ }^{1}$, R. Myers ${ }^{3}$, M. Brangier ${ }^{1}$, M. \\ Cohen $^{4}$, N. Dipper ${ }^{3}$, C. Evans ${ }^{5}$, D. Gratadour ${ }^{1}$, P. Jagourel ${ }^{4}$, P. Laporte ${ }^{4}$, D. Le Mignant ${ }^{6}$, M. Puech $^{4}$, \\ C. Robert ${ }^{2}$, H. Schnetler ${ }^{5}$, W. Taylor ${ }^{5}$, F. Vidal ${ }^{1}$, J.-G. Cuby ${ }^{6}$, M. Lehnert ${ }^{4}$, S. Morris ${ }^{3}$, and P. \\ Parr-Burmann 5 \\ 1 LESIA, Observatoire de Paris, Université Paris Diderot, 5 place J. Janssen, 92190 Meudon, France \\ 2 ONERA, 29 Av. de la division Leclerc, 92322, Châtillon, France \\ 3 Durham University, Department of Physics, South Road, Durham DH1 3LE, UK \\ 4 GEPI, Observatoire de Paris, Université Paris Diderot, 5 place J. Janssen, 92190 Meudon, France \\ 5 UK Astronomy Technology Centre, Blackford Hill, Edinburgh EH9 3HJ, UK \\ ${ }^{6}$ LAM, Technopôle de Marseille-Etoile, 38 rue F. Joliot-Curie, 13388 Marseille cedex 13
}

\begin{abstract}
EAGLE is the multi-object spatially-resolved near-IR spectrograph instrument concept for the EELT, relying on a distributed Adaptive Optics, so-called Multi Object Adaptive Optics. This paper presents the results of a phase A study. Using 84x84 actuator deformable mirrors, the performed analysis demonstrates that 6 laser guide stars and up to 5 natural guide stars of magnitude $R<17$, picked-up in a 7.3' diameter patrol field of view, allow us to obtain an overall performance in terms of Ensquared Energy of 35\% in a $75 \times 75 \mathrm{mas}^{2}$ spaxel at $H$ band whatever the target direction in the centred $5^{\prime}$ science field for median seeing conditions. The computed sky coverage at galactic latitudes $|b| \sim 60$ is close to $90 \%$.
\end{abstract}

\section{Introduction}

The European Extremely Large Telescope (E-ELT) is currently in phase B at ESO. This phase will end mid 2010 with the release of the proposal for the E-ELT construction. Meanwhile ESO has launched a number of instrument conceptual studies (phases A)[1]. A high priority instrument as derived from the science cases of the E-ELT, is a near IR spectrograph with multi, deployable Integral Field Units (IFUs), assisted by Adaptive Optics (AO). This type of instrument is particularly required for the study of the evolution of galaxies across cosmic times, addressing the key science areas on the physics and evolution of high-redshift galaxies, the detection and characterisation of first-light galaxies and the physics of galaxy evolution from stellar archaeology[2,3]. These are the main scientific drivers of EAGLE (Elt Adaptive optics for GaLaxy Evolution), the multi-object spatially-resolved near-IR spectrograph concept for the E-ELT [4]. EAGLE is a relatively simple instrument relying on a distributed AO concept, so-called MOAO (Multi Object AO). The EAGLE consortium consists of six institutes in France and in the UK. The EAGLE Phase A study started mid-2007 and ended in October 2009. But prototyping and demonstration activities will continue throughout 2010 and beyond. We present in this paper the phase A concept study of the AO system of this instrument.

\section{Instrument requirements and interfaces}

EAGLE is motivated by the desire to obtain near-IR spectroscopy of large numbers of objects across a wide field of view (FoV) of diameter $>5^{\prime}$, to build-up representative and unbiased samples of, for example, hundreds of high-redshift galaxies (see Ref. [4]). The requirement is to implement 20 IFUs in parallel and distributed in science FoV. It results from the trade-off between complexity/cost and observing time. The science case calls for improved angular resolution (75mas) with respect to the seeing but not diffraction-limited performance. The main scientific drivers are the improvement

\footnotetext{
a e-mail: gerard.rousset@obspm.fr
}

This is an Open Access article distributed under the terms of the Creative Commons Attribution-Noncommercial License, which permits unrestricted use, distribution, and reproduction in any noncommercial medium, provided the original work is properly cited. 


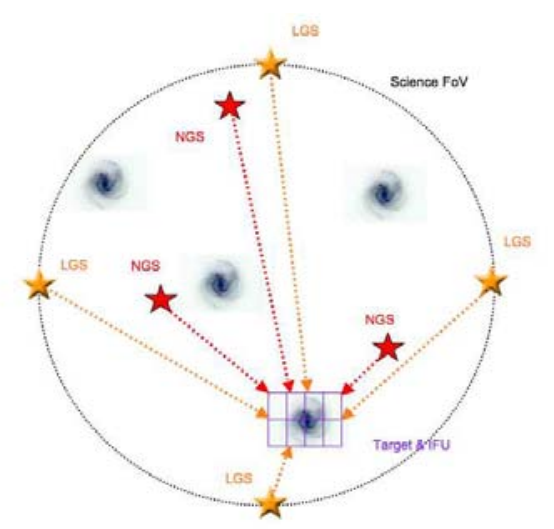

Fig. 1. MOAO configuration for LGS, NGS and science FoV ; IFU on target including a dedicated DM controlled in open loop through tomography using all the GS measurements (the arrows)

of the point source sensitivity and the ability to spatially resolve structures at the 100mas level. The performance requirement has therefore been set as 30 to $40 \%$ Ensquared Energy (EE) in a square spaxel of $75 \times 75 \mathrm{mas}^{2}$ in $H$ band $(1.6 \mu \mathrm{m})$. The subfield dedicated to each IFU is set to $1.65^{\prime \prime} \times 1.65^{\prime \prime}$ and sampled at 37.5mas. The wavelength coverage of the spectrographs extends from 0.8 to $2.5 \mu \mathrm{m}$.

The E-ELT baseline Design considers a 42-m telescope with a 5-mirror concept, mirrors active and adaptive. EAGLE is planned for installation at the Gravity Invariant Focal Station (GIFS) below the Nasmyth B platform allowing us to reduce significantly the problems associated with gravity induced flexure. The implementation contemplates a large retractable M6 mirror bending the full 10' diameter telescope FoV down to the GIFS. As a result of this model the instrument will need to take full control of the telescope, including the wavefront sensing Natural Guide Stars for controlling the telescope functions, such as co-phasing, telescope guiding and tracking, field stabilisation and active optics.

The first performance driver for the EAGLE AO is this Ensquared Energy (EE) requirement. The second one is the patrol FoV in the range of 5 to $10^{\prime}$ in diameter where the science targets are looking for and $\mathrm{EE}$ shall be achieved. Compensating for turbulence in such a wide FoV is a real challenge in AO. Despite it simplicity, Ground Layer AO will not achieve the EE requirement over the full patrol FoV considered for EAGLE [5]. Even Multi Conjugate AO (with a field segmentation) is not affordable due to the substantial increase of the number of deformable mirrors (DM) required to obtain the EE specification [5]. In fact, there is no need for a full correction of the entire FoV but rather only in the specific directions of the targets. Therefore, the Multi Object AO (MOAO) [6] is the only tractable concept allowing us to achieve the EE performance as demonstrated in this paper. As presented in Figure 1, the idea is to use one DM per direction of interest (target) in order to only compensate the IFU subfield of size $1.65^{\prime \prime} \times 1.65^{\prime \prime}$. For EAGLE, the science targets are too faint to allow any wavefront (WF) measurement. The correction to be applied to the science DMs is then computed by tomography from a set of guide stars (GS) distributed in the whole patrol FoV, on which the turbulent WFs are measured. These GS can be natural (NGS) or laser generated (LGS). Hence the control of the science DMs is done in open loop.

The first optical element of EAGLE in the telescope focal plane is a small 45 deg pick-off mirror to extract either the science target or the NGS, sending the light to the re-imaging optical train towards the IFU or the WF sensors (WFS), respectively. The science DMs are therefore implemented in the optical trains feeding the IFUs. The DM are conjugated to the telescope M4 mirror. Because of the large footprints of the LGS, they are extracted just above the focal plane with large pick-off mirrors, placed outside a preserved unvignetted central science FoV of $5^{\prime}$. The positions of the LGS are on an outer ring as depicted in Fig. 1. The EAGLE AO system will take advantage of E-ELT concept by controlling in closed loop the two in-telescope adaptive mirrors: M4 (deformable) and M5 (field stabilisation). It leads to a substantial reduction of the stroke required for the science DMs. 


\section{AO system analysis}

The relatively large spatial resolution element, when compared to that of a diffraction limited instrument (approximately ten times larger), has a huge influence on the design of AO and the error budget. Low Order (LO) modes (such as Tip-Tilt and defocus for instance) have very little impact on the overall performance of the system in terms of EE. On the other hand, the only way to improve EE is to correct the High Order (HO) spatial frequencies, requiring HO DMs and efficient tomographic reconstruction. For the numerical simulation, we use a WF power spectral density based tool [7,8] including multi-GS analysis, LGS and NGS WFSs, WF low order filtering for LGSs, optimal tomography reconstruction, dedicated FoV direction DM projection, point spread function (PSF) and EE computation. In this paper, the performance evaluation of EAGLE AO includes the tomographic error linked to the science FoV and the number and position of GSs, the propagated noise from the WFSs for both LGSs and NGSs, the fitting and aliasing effects due to the selected number of subapertures and actuators. The performance is EE, computed in 75x75mas from a set of PSFs at $H$ band, every 30 arc sec into the 5' diameter FoV.

A first analysis, presented elsewhere [5], lead to the requirements to use 9 LGSs for tomography, one NGS to measure the very low orders of the WF and high order science DMs. For cost and risk reduction purposes, we explored other configurations reducing the number of LGSs while taking advantage of the NGSs available in the FoV of the instrument. We investigate here the simultaneous variability of the following parameters:

- number of LGSs, 4 and 6 ;

- diameter of the FoV where NGSs could be picked-off, 5, 7.3 and 10'

- limiting magnitude of the NGSs for WF sensing, $R=15,17$ and 19 ;

- maximum number of available NGS-dedicated WFSs in the instrument, 1, 3 and 5.

We first assumed a seeing of $0.95 \mathrm{arcsec}$ at $0.5 \mu \mathrm{m}$ in the line of sight, an outer scale of $50 \mathrm{~m}$, ten turbulence layers between 0 and $16.5 \mathrm{~km}$, a low noise WF measurement variance of $0.1 \mathrm{rad}^{2}$ at $0.5 \mu \mathrm{m}$ for the LGSs, the filtering of the Zernike radial orders 1 and 2 on the LGSs, a noise WF measurement variance of $1 \mathrm{rad}^{2}$ at $0.5 \mu \mathrm{m}$ for a NGS of magnitude $R \simeq 11$ and a pupil sampling of 112 subapertures (for both LGS and NGS) and actuators in the diameter. For the 4 LGS case, EE in the central $5^{\prime}$ science FoV is only around $30 \%$ even considering 5 NGSs up to magnitude 19 in a patrol field of $10^{\prime}$. These values do not provide enough margin to include in the WF error (WFE) budget of the instrument all the other contributors not simulated here and therefore to be compliant with the specification. Another conclusion is that there is really a substantial gain in performance when increasing the patrol FoV to the largest possible value and increasing the number of available WFSs up to 5 because more NGSs are available to complement the measurements on the LGSs. But looking for NGSs up to $R=19$ does not bring any gain due to too low a signal to noise ratio for such NGSs in the WF measurements.

In Figure 2, we present the 6 LGS case considering a true cosmologic field of interest, the XMMLSS field as an example, taking into account the sky coverage issue linked to the availability of enough bright NGSs for the WF sensing. This field is located at a galactic latitude $b=-66.47 \mathrm{deg}$, we have star positions and magnitudes up to $R=19$. We chose 15 random positions within this field as the center of $5^{\prime}$ diameter science sub-fields and NGS pick-up fields. Fig. 2a shows the average, minimum and maximum EE for the 15 chosen sub-fields, assuming a NGS pick-up FoV limited to 7.3', 5 NGS dedicated WFSs, a NGS limiting magnitude of $R=17$. We observe a EE performance spread between 40 and $75 \%$ depending on the sub-field. We recall that considering only one bright NGS in the science field, we found $\mathrm{EE}=30 \%$ for $6 \mathrm{LGS}$ and $67 \%$ for 9 . In Fig. 2a, even if the variability of EE is important, it is possible most of the time to achieve EE greater or close to $50 \%$, slightly lower than the one with 9 LGS and one bright NGS, but a performance which could be sufficient depending on the total WFE budget. Fig. $2 b$ shows the sub-field 1 randomly selected in the XMM-LSS cosmologic field as an example corresponding to relatively poor performance in Fig. 2a. In the sub-field of 7.3' in diameter, only 4 NGS brighter than $R=17$ are available with only one relatively bright star $R=12.9$.

In terms of sky coverage, we analysed the number of NGS available in a given patrol field, for different regions of the sky using real target fields. For fields randomly selected at galactic latitudes of $50<|b|<90$, we obtain a chance larger than $80 \%$ to find five or more NGSs with $R<17$ in a $7^{\prime}$ 


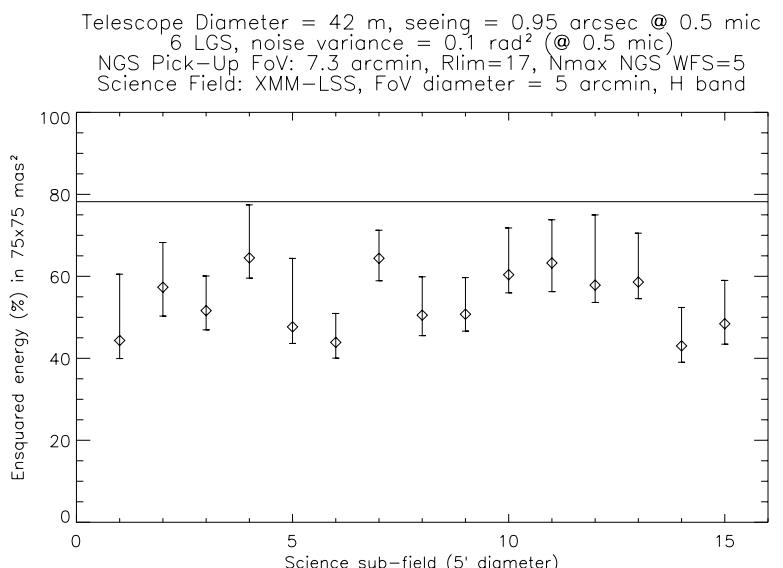

a

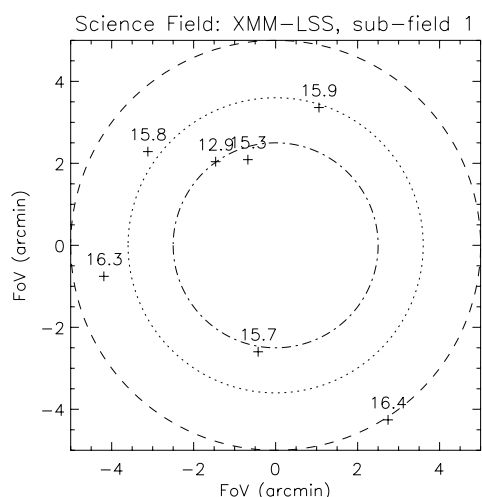

b

Fig. 2. [a] Simulated EE as a function of the randomly selected science sub-fields in the XMM-LSS cosmologic field: diamond average value and spread bar between min. and max. values computed on the whole $5^{\prime}$ sub-field. [b] One example of sub-fields. Crosses: NGS positions. Values: NGS magnitudes. Circle diameters: 10' for external, $7.3^{\prime}$ for intermediate, $5^{\prime}$ for internal.

diameter field. There is a considerable gain in NGS availability by exploiting patrol field beyond the central $5^{\prime}$ circular field. This result is also confirmed by star statistics. Using the Besancon model, we obtain a probability, to find five NGSs with $R<17$ in a 7.3' diameter field, larger than: $90 \%$ at latitude $|b|=60 \mathrm{deg}$ and $60 \%$ at galactic pole. For EAGLE, the strategy to take advantage of the large FoV to find around 5 NGSs for WF sensing is a good one, leading to attractive sky coverage, even at the galactic pole, and to the substantial reduction of the number of LGSs (9 to 6).

We decided also to consider median seeing conditions to finally define the number of actuators and subapertures. We assume a seeing of $0.87^{\prime \prime}$ at $0.5 \mu \mathrm{m}$ in the line of sight and an outer scale of $25 \mathrm{~m}$. We update the LGS measurement noise to be more realistic. In the simulation, we consider now an equivalent uniform variance for the WF measurement noise of $1 \mathrm{rad}^{2}$ at $0.589 \mu \mathrm{m}$. It is deduced from a fitting of the propagation through the tomographic reconstructor of fratricide effects and spot elongations, inducing non uniform noise in the pupil, for the case of a downscale telescope and the side launching $[9,10]$. This variance corresponds to around 500 photons per subaperture and per frame. For a subaperture of $50 \mathrm{~cm}$ in size and at $500 \mathrm{~Hz}$ frame rate, it typically corresponds to a launched power of $10 \mathrm{~W}$ per LGS. With these conditions, we analyse the choice of the number of actuators and WFS subapertures. The number of actuators on the science DMs is always equal to the number of subapertures of the LGS WFSs and varies between $64 \times 64,84 \times 84$ and $112 \times 112$. The number of NGS WFS subapertures is $32 \times 32$ and $64 \times 64$. Figure 3 shows the results of the simulation in terms of EE for these parameters for the sub-field 1, a worst case for the XMM-LSS field. It is found that in terms of tomographic and noise errors there is no substantial gain to go to $112 \times 112$ actuators and even with $64 \times 64$ the performance is quite impressive $\mathrm{EE}>50 \%$. For this field, there is a marginal gain to increase the number of subapertures for the NGS WFSs from $32 \times 32$ to $64 \times 64$ because 3 NGSs on the 4 available are faint, magnitude $R>15$. For more favorable cases, a typical $5 \%$ addition in EE is possible (for sub-field 10 for example). In conclusion, it is possible most of time to obtain with 6 LGS and up to 5 NGSs in a field of $7.3^{\prime}$ diameter a EE larger than $>50 \%$. We see that $84 \times 84$ are sufficient for the DMs and that $64 \times 64$ could be a back-up solution to the price of a slight decrease in performance. 


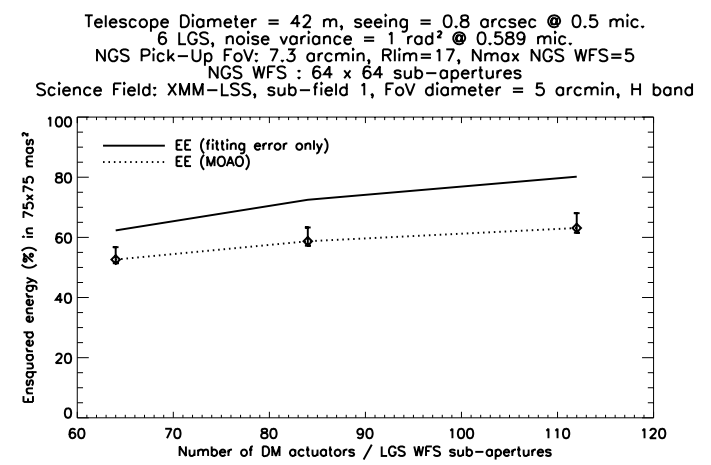

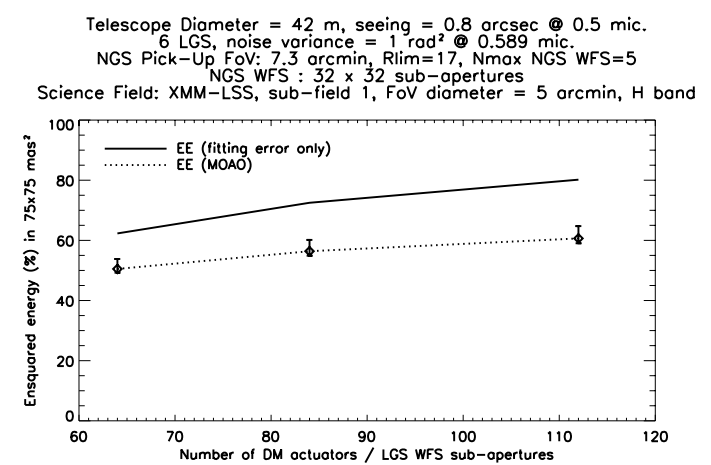

b

Fig. 3. Simulated EE as a function of the number of actuators (equal number of LGS WFS subapertures) for the sub-field 1 in the XMM-LSS cosmologic field (worst case): [a] 64x64 NGS WFS subapertures. [b] 32x32 NGS WFS subapertures. Diamond for average EE value and spread bar for min. and max. EE values, computed on the centred science $5^{\prime}$ field. Continuous line: fitting error only

\section{Preliminary AO system design}

The current design of the MOAO system for EAGLE is as followed. To ensure a good sky coverage and a relatively high performance, we propose to implement 6 LGSs of $10 \mathrm{~W}$ located on a 7.5' diameter ring around the science FoV. The LGS WFS will be Shack-Hartmann with 84x84 subapertures. The LGS detector is quite a challenge, but developments are conducted by ESO [11]. Up to 6 NGS WFSs of medium order ( $64 \times 64$ as a maximum) will be available to ensure the maximum covering of the $38.5 \operatorname{arcmin}^{2}$ patrol FoV. The NGSs will be picked-up anywhere in this FoV. The magnitude of these stars should be lower than $R=17$. The science channel DMs will be made of $84 \times 84$ actuators. The existing $4 k \mathrm{DM}$ should be a back-up solution in case of development problems for a larger count. The real time computer will implement an optimal tomographic reconstructor and a dedicated direction projector taking advantage of a $C_{n}^{2}$ profiling on site. The sampling temporal frequency of the loop should be ajustable between typically 250 and $25 \mathrm{~Hz}$. For calibrations, the main issue in addition to convention needs is the requirement of a very good pupil registration between all the parallel channels and its stability during observations because of the open loop constraints. We propose to use M4 (and some tools around to tag the pupil) to be able using artificial sources inside the telescope to record simultaneously and in a short time the current position of the image of M4 and the pupil conjugated components of the system. It will require to install in the science channels a WFS and a pupil imager to be used off-line only for this purpose.

The error budget of the AO system is split in LO and HO contributors having different impact on EE [5]. Dedicated expressions depending on the spatial frequencies allow us to link the WFE in $n m$ to the EE loss. In addition to the tomographic (including fitting and aliasing) and noise error, we take into account the following main errors: chromatism, refraction, differential focal anisoplanatism, temporal bandwidth, turbulence model, open loop both on the WFSs and on the DMs, calibrations and non common path aberrations, with some additional contingencies. Starting from the 53\% EE in the worst case given by the simulations presented in Section 3, it leads to an overall performance of $35 \%$ EE well in the specifications.

At system level, the MOAO is not yet tested on-sky, but there are several on-going programs worldwide which will demonstrate its principle of operations. Within the EAGLE framework, an aggressive technology development plan is in place which will allow us to demonstrate, test, and characterize MOAO within a couple of years. Demonstration activities are taking place on a laboratory test bench, SESAME, at Observatoire de Paris. Different DM technologies, such as magnetic, piezoelectric or electrostatic actuators, have been tested in terms of open loop behaviour. The laboratory measured open loop errors for a few DMs are compatible with the requirement of EAGLE: rms error of the order 
First conference on Adaptive Optics for Extremely Large Telescopes

of 2 to $4 \%$ of the rms stroke used for the compensation. The second key activity is the demonstration of the open loop optimised tomographic compensation [12]. The laboratory sumulation is in the conditions of a $4 \mathrm{~m}$ class telescope with 3 off-axis GSs, a piezoelectric DM in open loop in the on-axis diagnostic channel and a seeing corresponding to $D / r_{o}=12.8$. For a Strehl ratio of less than $1 \%$ without correction, we obtain 39\% in a conventional closed loop scheme and 35\% in pure open loop MOAO using an original tomography algorithm [12]. These results are the first stage improving the technology readiness. An on-sky demonstration programme (CANARY) is being actively developed for use at the WHT under the leadership of the Durham University [13], with the first results expected within 1 year with 3 off-axis NGSs and one on-axis channel for performance evaluation including 1 piezoelectric DM in open loop, and within 2 years with 4 additional Rayleigh LGSs, on time to validate the final EAGLE design.

\section{Conclusion}

The baseline design of EAGLE, the multi-object spatially-resolved near-IR spectrograph concept for the E-ELT, is driven by the scientific requirements to answer a number of crucial questions about how galaxies formed and evolved. MOAO is the only tractable AO concept achieving the specifications on the 20 parallel IFU subfields of size $1.65^{\prime \prime} \times 1.65^{\prime \prime}$ in a very wide FoV. Using a $84 \times 84$ actuator deformable mirror for each target direction, our analysis demonstrates that 6 laser guide stars and up to 5 natural guide stars of magnitude $R<17$, picked-up in a 7.3' diameter patrol field of view, are a good configuration for tomography. The computed performance is $\mathrm{EE}=35 \%$ in a $75 \times 75 \mathrm{mas}^{2}$ spaxel at $H$ band whatever the target direction in the centred $5^{\prime}$ science field, for median seeing conditions. The sky coverage at galactic latitudes $|b| \sim 60$ is close to $90 \%$ from both real fields and star statistics.

\section{Acknowledgements}

We acknowledge support from CNRS/INSU (France) and STFC (UK) ; the French Agence Nationale de la Recherche (ANR) program 06-BLAN-0191; the European Southern Observatory (ESO) (phase A study of a Wide Field, multi-IFU near IR Spectrograph and AO system for the E-ELT); the European Commission (Framework Programme 7, E-ELT Preparation, Infrastructure 2007-1 Grant 211257); the Observatoire de Paris.

\section{References}

1. Kissler-Patig M., in AO for ELTs, these proceedings, 2009

2. Puech M., Lehnert M., Yang Y. et al., in AO for ELTs, these proceedings, 2009

3. Evans C., Yang Y., Puech M. et al., in AO for ELTs, these proceedings, 2009

4. Cuby J.-G., Morris S., Parr-Burman P. et al., Proc. SPIE 7439, (2009) 0J1-0J12

5. Fusco T., Rousset G., Assemat F. et al., Proc. SPIE 7015, (2008) 0T1-0T12

6. Assemat F., Gendron E., Hammer F., MNRAS 376, (2007) 287-312

7. Neichel B., Fusco T. and Conan J.-M., J. Opt. Soc. Am. A 26, (2008) 219-235

8. Assemat F., Fusco T., Conan J.M. et al., Proc. SPIE 7015, (2008) 4A1-4A12

9. Robert C., Conan J.M., Gratadour D. et al., in AO for ELTs, these proceedings, 2009

10. Gratadour D., Gendron E., Rousset G. et al., in AO for ELTs, these proceedings, 2009

11. Kolb J., Downing M. and Clare R., in AO for ELTs, these proceedings, 2009

12. Vidal F., Gendron E., Brangier M. et al., in AO for ELTs, these proceedings, 2009

13. Morris T., Hubert Z., Myers R. et al., in AO for ELTs, these proceedings, 2009 